\title{
Using Deep Learning for Image-Based Potato Tuber Disease Detection
}

\author{
Dor Oppenheim, ${ }^{1, \dagger}$ Guy Shani, ${ }^{2}$ Orly Erlich, ${ }^{3}$ and Leah Tsror ${ }^{3}$ \\ ${ }^{1}$ Department of Industrial Engineering and Management, Ben-Gurion University of the Negev, Beer Sheva, Israel; ${ }^{2}$ Department of Software \\ and Information Systems Engineering, Ben-Gurion University of the Negev, Beer Sheva, Israel; and ${ }^{3}$ Department of Plant Pathology, Institute \\ of Plant Protection, Agricultural Research Organization, Gilat Center, M.P. Negev, Israel \\ Accepted for publication 8 December 2018.
}

\begin{abstract}
Many plant diseases have distinct visual symptoms, which can be used to identify and classify them correctly. This article presents a potato disease classification algorithm that leverages these distinct appearances and advances in computer vision made possible by deep learning. The algorithm uses a deep convolutional neural network, training it to classify the tubers into five classes: namely, four disease classes and a healthy potato class. The database of images used in this study, containing potato tubers of different cultivars, sizes, and diseases, was acquired, classified, and labeled manually by experts. The models were trained over different train-test splits to better understand the amount of image data needed to apply deep learning for
\end{abstract}

ABSTRACT

Potato (Solanum tuberosum) is the third most important food crop in the world, after cereals and rice. Global production exceeds 300 million metric tons and is an important nutrition and calorie provider for humanity (Pareek 2016). Potato production is threatened by many diseases resulting in considerable yield losses and/or a reduction in tuber quality, causing an increase of potato price (Taylor et al. 2008).

Potato tubers may be infected by various seed and soilborne pathogens that affect tuber quality and yield (Tsror et al. 1999). Among them are the fungal pathogens Helminthosporium solani, the causal agent of silver scurf; Colletotrichum coccodes, the causal agent of black dot; Rhizoctonia solani, the causal agent of black scurf; and the bacterial pathogen Streptomyces spp., the causal agent of common scab. All of these pathogens cause tuber blemishes that affect seed tubers as well as tubers for processing and for the fresh market trade (Mattupalli et al. 2013).

An accurate disease detection system in potato seeds is a crucial step for efficient disease management and can further prevent the spread of diseases (Rich 2013). Examination of tubers with a hand lens or microscope is required to observe the characteristic black microsclerotia of $C$. coccodes or the typical conidiophores and conidia of $H$. solani (Errampalli et al. 2001; Tsror and Peretz-Alon 2004). However, these structures are not always present, and additional diagnostics methods must be applied (e.g., isolation on selective media, serology, or molecular techniques). Manually detecting and sorting diseased potato tubers (either seed tubers or ware) is difficult, costly, and time-consuming, whereas computerized inspection may be more efficient and cost-effective.

†Corresponding author: D. Oppenheim; doropp@post.bgu.ac.il

Funding: This work is supported by the Ministry of Agriculture and partially supported by the Helmsley Charitable Trust through the Agricultural, Biological, and Cognitive Robotics Initiative and the Rabbi W. Gunther Plaut Chair in Manufacturing Engineering, both at Ben-Gurion University of the Negev.

The author(s) declare no conflict of interest.

(c) 2019 The American Phytopathological Society such classification tasks. The models were tested over a data set of images taken using standard low-cost RGB (red, green, and blue) sensors and were tagged by experts, demonstrating high classification accuracy. This is the first article to report the successful implementation of deep convolutional networks, popular in object identification, to the task of disease identification in potato tubers, showing the potential of deep learning techniques in agricultural tasks.

Keywords: Colletotrichum coccodes, Helminthosporium solani, image recognition, Rhizoctonia solani, Solanum tuberosum, Streptomyces spp., tuber blemish diseases.

Computer vision and machine learning techniques for disease detection have been broadly investigated in the last 2 decades (Barbedo 2016). Diseases can be detected using expensive and bulky digital imaging sensors, such as spectral or near-infrared sensors. Using such sensors encumbers the widespread implementation of these methods as a result of their high costs and maintenance (Sankaran et al. 2010). On the other hand, researchers using the visible light bandwidth, which can be captured by relatively low-cost cameras, have usually focused on a single type of disease (Zhang et al. 2014). A single case identification is insufficient for real-world applications, as a single tuber can be infected by a number of diseases (Cubero et al. 2016).

This article leverages advances in computer vision and object recognition for use in classifying multiple diseases in potato tubers. In 2012, a group of researchers from Toronto won the Large Scale Visual Recognition Challenge competition by improving the classification of the ImageNet database by more than $10 \%$. They achieved a top-five error rate of $15.3 \%$ when using a deep convolutional neural network (CNN), whereas the second best achieved a $26.2 \%$ error rate (Krizhevsky et al. 2012). Since then, CNN methods have improved and the classification error dropped to $3.73 \%$, which was achieved by the winning team for the same task (Abdi and Nahavandi 2016). In the field of computer vision for agricultural applications, the use of CNNs and other deep neural networks is continuously increasing (Gongal et al. 2015). A CNN was used for detecting and classifying seven fruit types in field conditions for agricultural tasks such as automated fruit picking, improving detection accuracy by $3 \%$ over previous methods (Sa et al. 2016). CNNs used in classification tasks, such as classification of disease symptoms on plant leaves or quality control of harvested fruit and vegetables, reached accuracy of more than $97 \%$ (Mohanty et al. 2016; Tan et al. 2016). To create successful CNNs, a large amount of training data are needed (Sermanet et al. 2013). Therefore, the first aim of the current research was to identify selected potato tuber disease-based $\mathrm{CNNs}$ after acquiring a collection of a sufficient data set and classification of the displayed diseases. The results indicate a first step toward multiple disease classification for potatoes using CNNs. 


\section{MATERIALS AND METHODS}

Data acquisition. Photos of 400 diseased potato tubers with smooth skin but different cultivars, shapes, sizes, and colors were taken under normal uncontrolled illumination conditions. The tubers were manually classified by potato pathology experts as a standard procedure of monitoring the incidence and severity of various diseases in seed potato tubers prior to planting. This procedure is done annually independent of the current research, with both seed lots imported from Europe to Israel for the spring season and domestic seed lots for the winter season (Tsror et al. 1999). The potato tubers were contaminated with several diseases simultaneously; however, for the current research, tubers were selected with four different diseases (separately), all with significant and typical symptoms on the tuber skin (Fig. 1). The images were acquired using multiple types of standard cameras, captured from one viewpoint only. We choose to use a variety of simple low-cost RGB (red, green, and blue) sensors found in cell phones and simple digital cameras to show the robustness of our methods to variations in the image sensor.

Data preparation. The images acquired were used to create the training and tests sets for the CNN. Every visual symptom of a disease was marked and labeled using the image labeler application in MatLab 2014b software. The labeling was done with rectangular bounding boxes encompassing the visual symptom but also additional healthy potato skin, as seen in Figure 2. The marked areas were cropped from the original image, transformed into grayscale, and resized to a standard 224- $\times 224$-pixel square. After preprocessing, a total of 2,465 patches of diseased potato skin were gathered, including 265 black dot patches, 469 black scurf patches, 686 common scab patches, 738 silver scurf patches, and 307 uninfected patches.

Performance measurement. The experiment was designed to evaluate the performance of the CNN's learning algorithm in classifying four diseases and visually symptom-free potatoes. Because manually labeling diseased patches of potatoes is a tedious

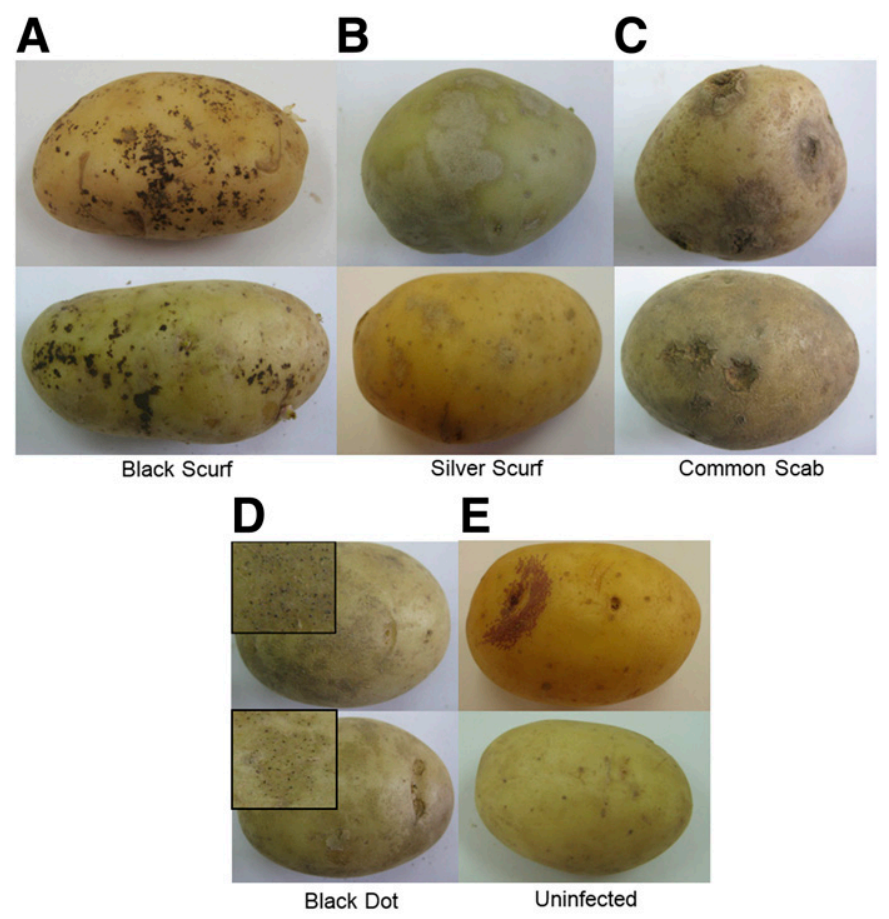

Fig. 1. Examples of visual symptoms of potato diseases. A, Black scurf: irregular, black, scab-like marks on the skin of the tuber. B, Silver scurf: circular or irregular, tan to silvery gray lesions on the tuber's skin. $\mathbf{C}$, Common scab: circular brown rough areas with irregular margins that can coalesce into larger areas. D, Black dot: tiny black dots on the skin of the tuber (hardly visible in small images). E, Uninfected tuber. and time-costly task, an important task was to determine the minimal amount of training data that provide sufficient classification accuracy. The CNN was trained with different sizes of training sets. The smallest training set used for training was $10 \%$ of the 2,465 images, which increased incrementally by 10 to $90 \%$ of the whole data set as detailed in Table 1 . In each increment, the images were selected randomly from the whole data set. Testing of the algorithm was done on the remaining data. In total, the training and testing phases were repeated nine times over different training set sizes.

Each training set was trained for 90 epochs, in which one epoch is defined as one full training cycle on every sample in the training set. The choice of limiting to 90 epochs was made based on empirical observation that the learning process converged well within 90 epochs (Fig. 3). To compare between the different results over the nine training sets, the error rate of the best scoring guess was calculated as the number of errors divided by the total number of test images in every epoch. The error was calculated for both the test and train sets in order to understand the over- and underfitting of the procedure.

Algorithm. The algorithm chosen for the image classification task was a deep CNN. The basic architecture chosen for this problem was a CNN developed by the Visual Geometry Group (VGG) from the University of Oxford, named CNN-F because of its faster training time (Chatfield et al. 2014). Several new dropout layers were added to the VGG architecture to address problems of overfitting, especially as a result of the relatively small data set. The required input image size for this network is a $224 \times 224$ matrix. The CNN comprises eight learnable layers, the first five of which are convolutional, followed by three fully connected layers and ending with a softmax layer (Fig. 4). The softmax layer normalizes the input received from the final fully connected layer (fc3), producing a distribution of values (one for each class). The sum of these values adds up to 1 and they represent the probability of the input image to belong to one of the five classes. This softmax layer was also altered and adapted, reducing its size from 1,000 to 5 to fit our classification task. Training CNNs usually requires a large amount of labeled data in order to perform a good classification. Therefore, two methods were used for data augmentation. First, mirroring creates additional examples by flipping the images used in training randomly. Because the direction of the photos was arbitrary, mirroring the image horizontally did not change the correctness of the data. Second, cropping was also used. Cropping the image randomly to different sizes, while keeping the cropped image minimum size to $190 \times 190$, can achieve data diversity. The use of each data augmentation method was done randomly. Before each image was inserted into the net for training, it was mirrored, cropped, or inserted without altering in equal distributions. Therefore, two-thirds of the images trained were altered.

\section{RESULTS}

Figure 5 shows the confusion matrix of the identification accuracy for a model that trained over $90 \%$ of the data. The confusion matrix's columns represent the CNN's class classification, whereas the rows represent the actual classes. This type of representation can help evaluate the CNN's classification of each class. As can be seen, there were no errors for black dots and black scurf, which have very distinctive visual symptoms. Not surprisingly, there is some confusion between silver scurf and common scab, which can also seem similar to an inexperienced human observer. The performance of the CNN was lower when classifying visually healthy tubers. Most of the CNN's misclassifications occurred when classifying these visually healthy tubers to the disease class of silver scurf. Silver scurf's visual symptoms are bright tan to silvery gray lesions on the tuber's skin, which may resemble uninfected skin.

Because deep learning often requires training with many samples, we also evaluated the overall accuracy with respect to the amount of training data. Table 2 shows the accuracy as a function 
of the amount of training data. The best trained model (trained on $90 \%$ of the data set and tested on the remaining 10\%) correctly classified $96 \%$ of the images. Not surprisingly, more data increase performance. However, beyond $60 \%$ of the data, the increase in performance is minor.

Deep learning models are also notorious for requiring much training time. Figure 3 shows the accuracy as a function of the amount of training epochs. As can be seen, the performance drastically improves over the first 25 epochs, but improvement is minor beyond 50 epochs. Figure 3 also compares the amount of training data and shows that with $10 \%$ of the data, the learning process is quite noisy; however, with more data, the learning curve becomes smoother and more consistent.

\section{DISCUSSION}

The results above indicate, as expected, that using more data for the training phase improves the classification and reduces the error

\section{A}

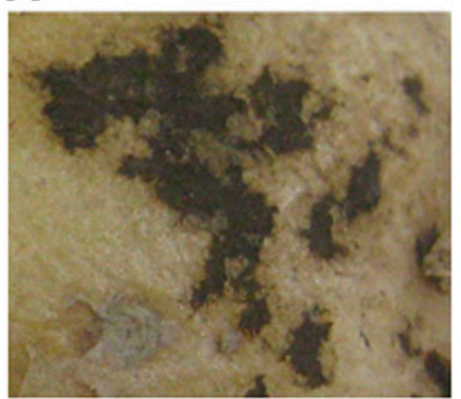

Black Scurf patch: color

D

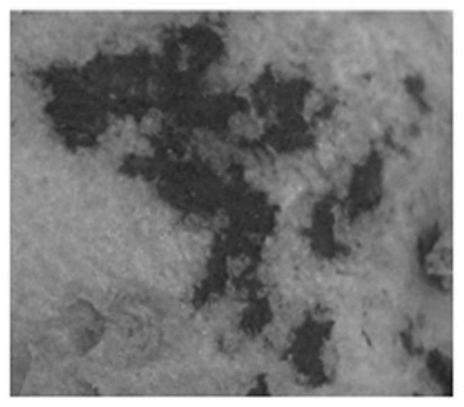

Black Scurf patch: grayscale

G

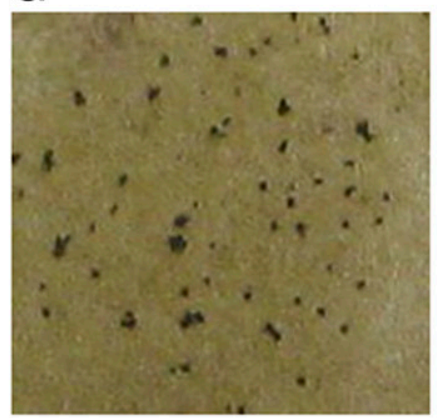

Black Dot patch: color

I

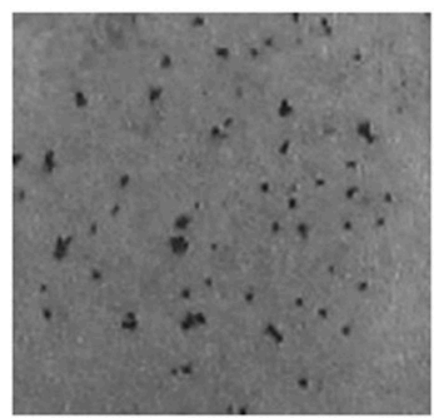

Black Dot patch: grayscale

B

$\mathbf{E}$

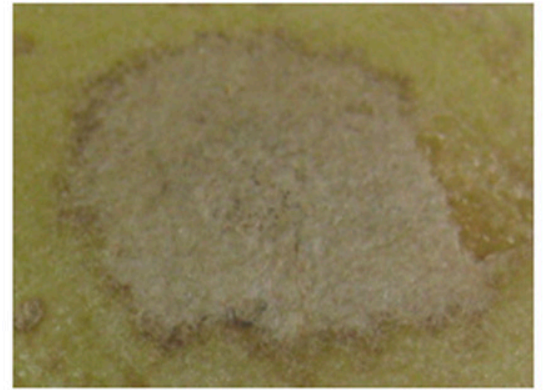

Silver Scurf patch: color

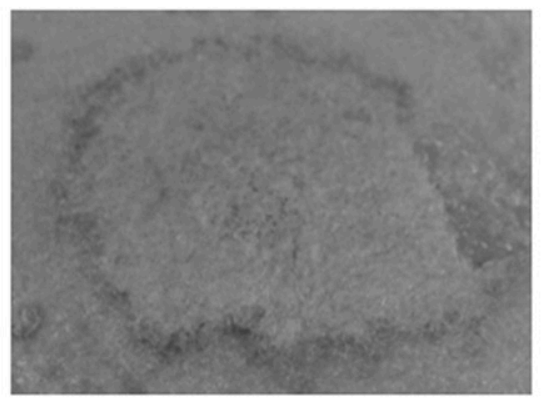

Silver Scurf patch: grayscale

H

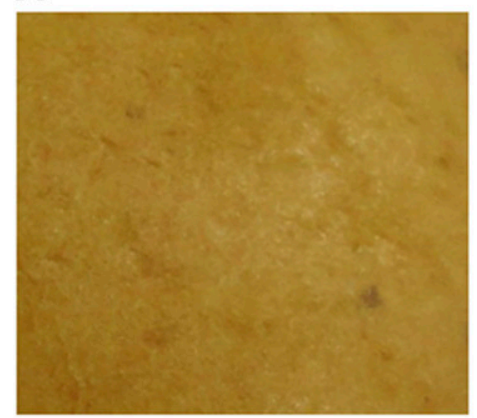

Uninfected patch: color

J

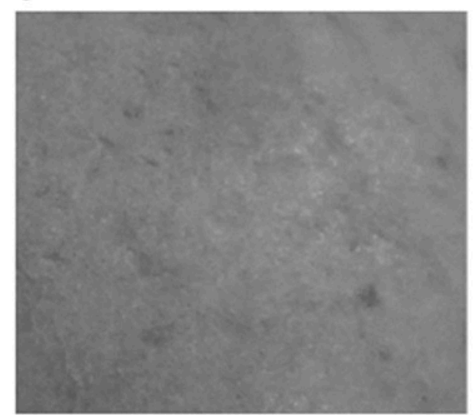

Uninfected patch: grayscale

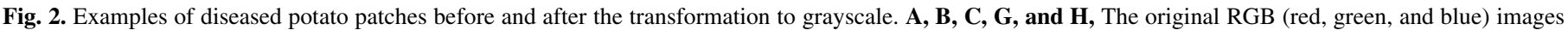
from each class. D, E, F, I, and J, The same images after the conversion to grayscale using Matlab's rgb2gray function. 
rate. The relatively small decrease in accuracy (Table 2) for most training set sizes is an indicator that a small number of potato images could suffice for training a sufficiently accurate CNN.

The results also show that the trained CNN can correctly and accurately classify the four diseases presented here. However, visually healthy tubers were harder to classify. The fact that the diseases were classified with high accuracy makes the CNN suitable for a system in which identification of the disease is important. Most misclassifications occurred for the visually healthy class. Arguably, false-positive mistakes (classifying a healthy tuber as diseased) are less problematic than false-negative mistakes (classifying a diseased tuber as healthy), because planting infected tubers can spread disease and cause considerable damage. On the other hand, avoiding planting a small number of healthy tubers may not pose a practical problem. In this experiment, only 307 images of visually healthy tubers were used; increasing the amount of data on uninfected tubers can increase the classification accuracy of this class.

Although increasing the size of the training set can be helpful, the problem might persist as a result of an imbalanced set of images. This is because some diseases are much more common than others. In such cases, classification algorithms often ignore the less frequent class. Several algorithms were suggested for handling imbalanced data sets, especially in the case of training CNN models (Dong et al. 2018; Masko and Hensman 2015). An investigation of such methods is left for future research.

TABLE 1. Best-performing model accuracy results for each train-test set

\begin{tabular}{lcc}
\hline Train-test set split portion $(\%)$ & Train-test set split quantity & Accuracy \\
\hline 90 to 10 & $360-40$ & 0.9585 \\
80 to 20 & $320-80$ & 0.9567 \\
70 to 30 & $280-120$ & 0.9465 \\
60 to 40 & $240-160$ & 0.9454 \\
50 to 50 & $200-200$ & 0.9069 \\
40 to 60 & $160-20$ & 0.9183 \\
30 to 70 & $120-280$ & 0.9041 \\
20 to 80 & $80-320$ & 0.9012 \\
10 to 90 & $40-360$ & 0.8321 \\
\hline
\end{tabular}

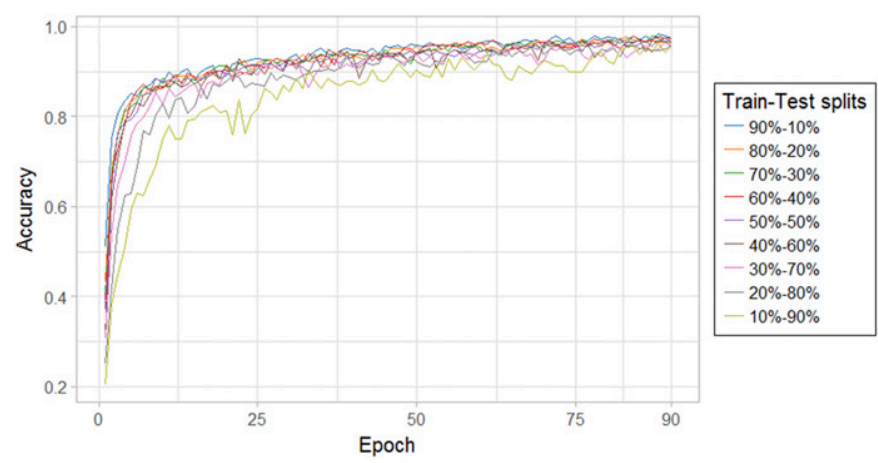

Fig. 3. Results of experiment. Plot shows the accuracy of each training set (Shown as different colors on graph) according to the epoch number.
Automated detection of potato disease from sensor output has previously been suggested. Sankaran et al. (2010) review methods for automated disease detection in plants, arguing that there is a need for a low-cost, fast, and reliable health monitoring system. They report techniques that use florescence images taken using a halogen lamp as an ultraviolet light source. Measuring at different light frequency can be helpful, mostly in leaves. Hyperspectral techniques that measure multiple wavelengths were also found useful in disease detection, as some symptoms are easily detected in certain wavelengths. Studies on potatoes tubers were performed using volatile organic compound (VOC) release (de Lacy Costello et al. 2001; Martinelli et al. 2015), rather than by digital images.

In cases in which it is critical to avoid all false-negative results, it may well be that the CNN model can be used as a first screening phase. Then all tubers suspected as infected can be examined using more accurate, yet costly, methods such as VOCs. This may apply in the screening of seed tubers to avoid the spread of disease. CNNs may suffice for other applications such as in field identification by farmers after planting, in which accuracy can be compromised for reduced costs.

Dacal-Nieto et al. (2011) reported on the detection of common scab using hyperspectral images. They applied several machine learning classification techniques, including support vector machines (SVMs) and random forests over a set of 234 potato tubers with different degrees of common scab, which is a much smaller set than we collected. They also did not experiment with classifying tubers with different diseases. They reported an accuracy of $97.1 \%$ for the best variant. We achieved a lower accuracy of $92.56 \%$, mainly owing to confusion with silver scurf, which has relatively similar visual symptoms. To note, hyperspectral imaging sensors are very costly, and they produce much more information than the standard RGB sensors we used. On the other hand, the significant leap forward in object detection using deep learning occurred after

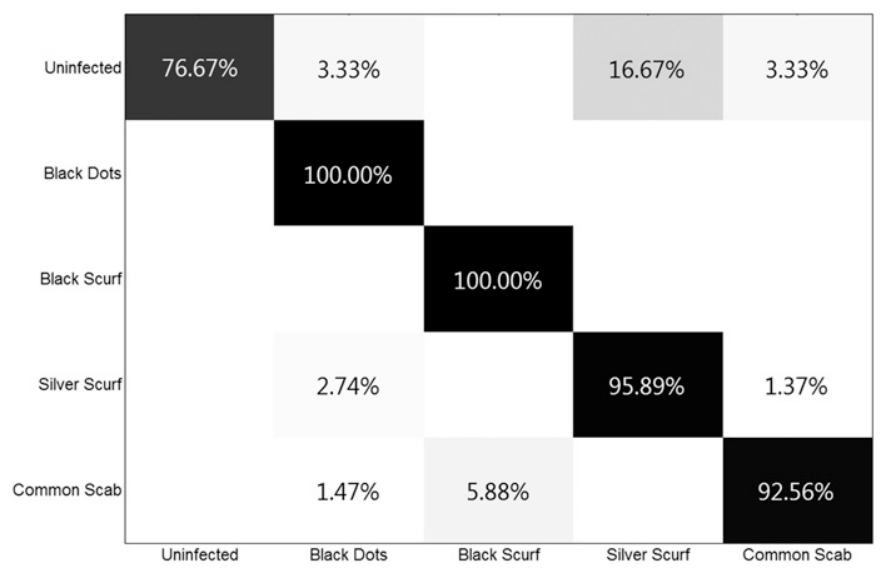

Fig. 5. A confusion matrix of the convolutional neural network $(\mathrm{CNN})$ trained on $90 \%$ of the data set and tested on the remaining $10 \%$. Rows represent the actual classes of an image. Columns represent the CNN's class prediction. Each cell in the matrix represents the percentage of images of the row's class that were classified to the column's class.

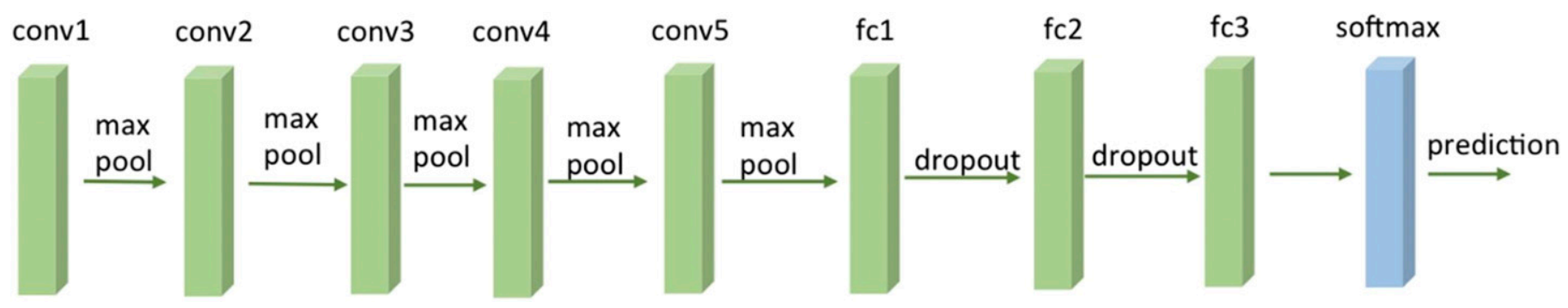

Fig. 4. A simplified model of the convolutional neural network (CNN). 
TABLE 2. Train and test set division for image patches

\begin{tabular}{lccccc}
\hline $\begin{array}{l}\text { Train-test split } \\
(\%)\end{array}$ & $\begin{array}{c}\text { Black } \\
\text { scurf }\end{array}$ & $\begin{array}{c}\text { Common } \\
\text { scab }\end{array}$ & $\begin{array}{c}\text { Silver } \\
\text { scurf }\end{array}$ & $\begin{array}{c}\text { Black } \\
\text { dot }\end{array}$ & $\begin{array}{l}\text { Visually } \\
\text { healthy }\end{array}$ \\
\hline 90 to 10 & $423 / 46$ & $618 / 68$ & $665 / 73$ & $239 / 26$ & $277 / 30$ \\
80 to 20 & $377 / 92$ & $550 / 136$ & $592 / 146$ & $213 / 52$ & $247 / 60$ \\
70 to 30 & $331 / 138$ & $482 / 204$ & $519 / 219$ & $187 / 78$ & $217 / 90$ \\
60 to 40 & $285 / 184$ & $414 / 272$ & $446 / 292$ & $161 / 104$ & $187 / 120$ \\
50 to 50 & $239 / 230$ & $346 / 340$ & $373 / 365$ & $135 / 130$ & $157 / 150$ \\
40 to 60 & $193 / 276$ & $278 / 408$ & $300 / 438$ & $109 / 156$ & $127 / 180$ \\
30 to 70 & $147 / 322$ & $210 / 476$ & $227 / 511$ & $83 / 182$ & $97 / 210$ \\
20 to 80 & $101 / 368$ & $142 / 544$ & $154 / 584$ & $57 / 208$ & $67 / 240$ \\
10 to 90 & $55 / 414$ & $74 / 612$ & $81 / 657$ & $31 / 234$ & $37 / 270$ \\
Total & 469 & 686 & 738 & 265 & 307 \\
\hline
\end{tabular}

the time of the study by Dacal-Nieto et al. (2011) and the combination of hyperspectral images and algorithms, such as the faster R-CNN used in this study, may have yielded better accuracy.

Hallau et al. (2018) discussed disease identification in sugar beets using standard RGB cameras, as done in this study. They focused on the different task of identifying visual symptoms on leaves, rather than on the tuber, as done here. Hallau et al. (2018) did not use deep learning object detection; rather, they used SVMs with a set of problem-specific features, and they reported an accuracy of about $75 \%$ in classifying between six different diseases.

Mohanty et al. (2016) suggested using deep learning CNN methods for disease identification in plants. They used a large set of 54,306 images of 38 classes of different plants and diseases, including potatoes with early and late blight. They reported accuracy between 85 and $99 \%$ on the different classes. The task they used is somewhat simpler, as identifying different plants may be easier than identifying different diseases on the same plant. Yet their work points to the power of deep learning in image identification in agriculture. Similar work was done by Fuentes et al. (2017) using the faster R-CNN algorithm that we also employed. They experimented with tomato leaf images displaying visual symptoms generated by 10 different tomato diseases and pests. They reported similar accuracy results to those shown here, higher than $90 \%$.

Conclusions. The applicability of a CNN for the task of classifying image patches of diseased potato tubers into four disease classes and a visually healthy class was examined. A set of 2,465 images of diseased patches was collected using a variety of low-cost RGB sensors under uncontrolled light conditions. These images were classified by a trained $\mathrm{CNN}$ model. The results indicate the robustness of the classification algorithm allowing for uncontrolled acquisition conditions. The results reveal that the correct classification of fully trained CNN models ranges from $83 \%$ for the model trained on the least amount of data, to $96 \%$ for the model trained on $90 \%$ of the data. To obtain classification rates higher than $90 \%$, it is sufficient to use 493 images from our data set. Our results on image patches suggest that combining a sliding window algorithm or an object detection network, such as faster R-CNN (Ren et al. 2015), can facilitate disease detection in images of complete tubers. Ongoing research aims to develop a classification algorithm with an expanded number of disease classes. Data can be acquired easily because there are no constraints on data acquisition.

\section{ACKNOWLEDGMENTS}

We thank Y. Edan for her important comments.

\section{LITERATURE CITED}

Abdi, M., and Nahavandi, S. 2016. Multi-residual networks: Improving the speed and accuracy of residual networks. arXiv 1609.05672.

Barbedo, J. G. A. 2016. A review on the main challenges in automatic plant disease identification based on visible range images. Biosyst. Eng. 144:52-60.

Chatfield, K., Simonyan, K., Vedaldi, A., and Zisserman, A. 2014. Return of the devil in the details: Delving deep into convolutional nets. arXiv 1405.3531.
Cubero, S., Lee, W. S., Aleixos, N., Albert, F., and Blasco, J. 2016. Automated systems based on machine vision for inspecting citrus fruits from the field to postharvest-a review. Food Bioprocess Technol. 9:1623-1639.

Dacal-Nieto, A., Formella, A., Carrión, P., Vazquez-Fernandez, E., and Fernández-Delgado, M. 2011. Common scab detection on potatoes using an infrared hyperspectral imaging system. Pages 303-312 in: International Conference on Image Analysis and Processing. Springer, Berlin, Germany.

de Lacy Costello, B. P. J., Evans, P., Ewen, R. J., Gunson, H. E., Jones, P. R., Ratcliffe, N. M., and Spencer-Phillips, P. T. 2001. Gas chromatographymass spectrometry analyses of volatile organic compounds from potato tubers inoculated with Phytophthora infestans or Fusarium coeruleum. Plant Pathol. 50:489-496.

Dong, Q., Gong, S., and Zhu, X. 2018. Imbalanced deep learning by minority class incremental rectification. IEEE Trans. Pattern Anal. Mach. Intell. Online publication. doi.org/10.1109/TPAMI.2018.2832629

Errampalli, D., Saunders, J. M., and Holley, J. D. 2001. Emergence of silver scurf (Helminthosporium solani) as an economically important disease of potato. Plant Pathol. 50:141-153.

Fuentes, A., Yoon, S., Kim, S. C., and Park, D. S. 2017. A robust deeplearning-based detector for real-time tomato plant diseases and pests recognition. Sensors (Basel) 17:2022.

Gongal, A., Amatya, S., Karkee, M., Zhang, Q., and Lewis, K. 2015. Sensors and systems for fruit detection and localization: A review. Comput. Electron. Agric. 116:8-19.

Hallau, L., Neumann, M., Klatt, B., Kleinhenz, B., Klein, T., Kuhn, C., Röhrig, M., Bauckhage, C., Kersting, K., and Mahlein, A.-K. 2018. Automated identification of sugar beet diseases using smartphones. Plant Pathol. 67:399-410.

Krizhevsky, A., Sutskever, I., and Hinton, G. E. 2012. ImageNet classification with deep convolutional neural networks. Pages 1097-1105 in: Advances in Neural Information Processing Systems 25. F. Pereira, C. J. C. Burges, L. Bottou, and K. Q. Weinberger, eds. Neural Information Processing Systems Foundation Inc., La Jolla, CA.

Martinelli, F., Scalenghe, R., Davino, S., Panno, S., Scuderi, G., Ruisi, P., Villa, P., Stroppiana, D., Boschetti, M., Goulart, L. R., and Davis, C.E. 2015. Advanced methods of plant disease detection. A review. Agron. Sustain. Dev. 35:1-25.

Masko, D., and Hensman, P. 2015. The Impact of Imbalanced Training Data for Convolutional Neural Networks. Thesis, KTH Royal Institute of Technology, Stockholm, Sweden.

Mattupalli, C., Genger, R. K., and Charkowski, A. O. 2013. Evaluating incidence of Helminthosporium solani and Colletotrichum coccodes on asymptomatic organic potatoes and screening potato lines for resistance to silver scurf. Am. J. Potato Res. 90:369-377.

Mohanty, S. P., Hughes, D. P., and Salathé, M. 2016. Using deep learning for image-based plant disease detection. Front. Plant Sci. 7:1419.

Pareek, S. 2016. Postharvest Ripening Physiology of Crops, Vol. 1. CRC Press, Boca Raton, FL.

Ren, S., He, K., Girshick, R. and Sun, J. 2015. Faster R-CNN: Towards realtime object detection with region proposal networks. Pages 91-99 in: Advances in Neural Information Processing Systems 28. C. Cortes, N. D. Lawrence, D. D. Lee, M. Sugiyama, and R. Garnett. eds. Neural Information Processing Systems Foundation Inc., La Jolla, CA.

Rich, A. E. 2013. Potato Diseases. Academic Press, New York.

Sa, I., Ge, Z., Dayoub, F., Ben Upcroft, T. P., and McCool, C. 2016. Deepfruits: A fruit detection system using deep neural networks. Sensors (Basel) 16:1222.

Sankaran, S., Mishra, A., Ehsani, R., and Davis, C. 2010. A review of advanced techniques for detecting plant diseases. Comput. Electron. Agric. 72:1-13.

Sermanet, P., Eigen, D., Zhang, X., Mathieu, M., Fergus, R., and LeCun, Y. 2013. Overfeat: Integrated recognition, localization and detection using convolutional networks. arXiv 1312.6229.

Tan, W., Zhao, C., and Wu, H. 2016. Intelligent alerting for fruit-melon lesion image based on momentum deep learning. Multimedia Tools Appl. 75: 16741-16761.

Taylor, R. J., Pasche, J. S., and Gudmestad, N. C. 2008. Susceptibility of eight potato cultivars to tuber infection by Phytophthora erythroseptica and Pythium ultimum and its relationship to mefenoxam-mediated control of pink rot and leak. Ann. Appl. Biol. 152:189-199.

Tsror, L., Erlich, O., and Hazanovsky, M. 1999. Effect of Colletotrichum coccodes on potato yield, tuber quality, and stem colonization during spring and autumn. Plant Dis. 83:561-565.

Tsror, L., and Peretz-Alon, I. 2004. Control of silver scurf on potato by dusting or spraying seed tubers with fungicides before planting. Am. J. Potato Res. 81:291-294.

Zhang, B., Huang, W., Li, J., Zhao, C., Fan, S., Wu, J., and Liu, C. 2014. Principles, developments and applications of computer vision for external quality inspection of fruits and vegetables: A review. Food Res. Int. 62: 326-343. 